\title{
Tagungsbericht
}

\author{
Ina Blümel*, Silke Clausing, Franziska Altemeier, Amelie Andresen, Aline Brun, Carina Dony, \\ Marisa Kaminski, Antje Pellowski, Helena Schugt und Anne Talk
}

\section{Familiär im Norden - Nachlese zur 26. BOBCATSSS in Riga, Lettland}

https://doi.org/10.1515/iwp-2018-0024

Bereits zum dritten Mal in Folge nahm eine zehnköpfige Gruppe Studierender der Hochschule Hannover aus dem Vollzeit ${ }^{1}$ sowie dem berufsbegleitenden ${ }^{2}$ Bachelor-Studiengang Informationsmanagement, in Begleitung zweier Dozentinnen, an der BOBCATSSS-Konferenz teil. Nachdem bisher ausschließlich auf der Hochschulwebseite und tagesaktuell im Blog ${ }^{3}$ über Erfahrungen und Erlebnisse während der drei BOBCATSSS-Exkursionen berichtet wurde, folgt hiermit nun ein zusammenhängender Beitrag über die diesjährige Konferenz vom 24. bis zum 26. Januar 2018 in Riga, Lettland.

Zum Hintergrund: Die BOBCATSSS-Konferenz ist eine durchaus traditionsreiche - sie jährt sich bereits zum 26 . Mal - internationale Konferenz der Bibliotheks- und Informationswissenschaften. Im Gegensatz zu IFLA \& Co wird sie fast ausschließlich von Studierenden wechselnder Universitäten in Europa ausgerichtet, und das sehr professionell. So sind auch die Beitragenden und Konferenzteilnehmer vornehmlich LIS-Studierende und junge Absolventen,

1 vgl. Informationsmanagement - Hochschule Hannover. Online verfügbar unter http://im.f3.hs-hannover.de/ [17.2.2018].

2 vgl. Hochschule Hannover: Studiengang Informationsmanagement - berufsbegleitend. Online verfügbar unter https://f3.hs-hann over.de/studium/bachelor/informationsmanagement-berufsbegle itend/index.html [17.2.2018].

3 vgl. Exkursionen und Internationale Kurzprogramme. Online verfügbar unter https://f3international.wordpress.com/ [17.2.2018].

*Kontaktperson: Dr. Ina Blümel,

E-Mail: ina.bluemel@hs-hannover.de

Silke Clausing, E-Mail: silke.clausing@hs-hannover.de Franziska Altemeier,

E-Mail: franziska.altemeier@stud.hs-hannover.de

Amelie Andresen, E-Mail: amelie.andresen@stud.hs-hannover.de

Aline Brun, E-Mail: aline.brun@stud.hs-hannover.de

Carina Dony, E-Mail: carina.dony@stud.hs-hannover.de

Marisa Kaminski, E-Mail: marisa.kaminski@stud.hs-hannover.de

Antje Pellowski, E-Mail: antje.pellowski@stud.hs-hannover.de

Helena Schugt, E-Mail: helena.schugt@stud.hs-hannover.de

Anne Talk, E-Mail: anne.talk@stud.hs-hannover.de ebenfalls die Session-Chairs, die Jury, usw. Einzig die Keynote-Speaker kommen nicht aus den Reihen der Young Professionals. Workshops und Paper Sessions stehen wie bei den „älteren“ Konferenzvorbildern im Mittelpunkt der BOBCATSSS. Sie behandeln Fragestellungen zu den jährlich wechselnden Themenschwerpunkten und dienen dem fachlichen Austausch und internationalen Netzwerken von Studierenden, ihren Lehrenden, Forschenden und anderen Interessierten. An der Erstausrichtung 1993 waren die Universitäten in Budapest, Oslo, Barcelona, Kopenhagen, Amsterdam, Tampere, Stuttgart, Szombathely und Sheffield beteiligt. In Anlehnung hieran entwickelte sich das Akronym BOBCATSSS. Während der letzten Jahre sind weitere - insbesondere osteuropäische - Städte hinzugekommen. Die Konferenz steht unter der Schirmherrschaft der EUCLID (European Association for Library and Information Education and Research).

Die 26. BOBCATSSS-Konferenz stand unter dem Motto „The Power of Reading” und wurde von der Latvijas Universitātē in Kooperation mit der Eötvös Loránd Tudományegyetem (University of Budapest) organisiert. ${ }^{4}$ Drei Keynote-Vorträge betrachteten das Thema aus unterschiedlichen Blickwinkeln. Prof. Dr. Jurǵis Škilters, Leiter des Labors für Wahrnehmungs- und Erkennungssysteme an der Latvijas Universitāte in Riga, berichtete von seinen Forschungen dazu, wie Menschen Zeichen und Symbole optisch wahrnehmen und wie diese Wahrnehmung wissenschaftlich eruiert werden kann. Zudem ging er auf das sich ändernde Leseverhalten ein, das mit dem Wechsel von Print- zu digitalen Quellen einhergeht. Frau Prof. Dr. Ute Schneider, akademische Direktorin der Abteilung Buchwissenschaft am Gutenberg-Institut für Weltliteratur und schriftorientierte Medien in Mainz, stellte den Leser in den Fokus ihrer Keynote und betrachtete die Selbstdarstellung des persönlichen Leseverhaltens in sozialen Netzwerken, wie Instagram und YouTube. Frau Dr. Katalin Bella

4 vgl. BOBCATSSS 2018. Online verfügbar unter http://bobcatsss 2018.lu.lv/ [17.2.2018]. 


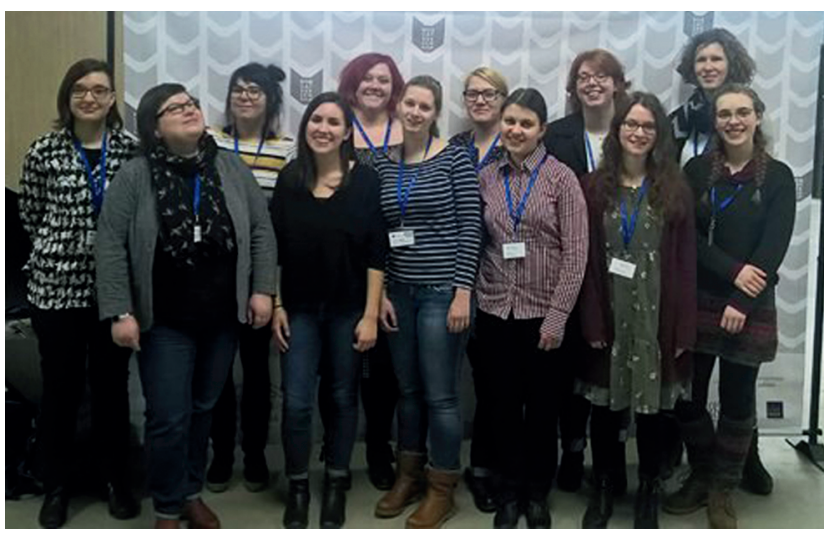

Abbildung 1: Gruppenfoto HSH-Gruppe (vlnr: Antje Pellowski, Silke Clausing, Franziska Altemeier, Aline Brun, Mareike Theis, Marisa Kaminski, Anne Talk, Carina Dony, Helena Schugt, Alina Balandis, Ina Blümel, Amelie Andresen). (Foto: Ina Blümel)

vom Institut für Bibliotheks- und Informationswissenschaften der Eötvös Loránd Universität in Budapest/Ungarn referierte über die persönlichen Lesegewohnheiten der wichtigsten ungarischen Autoren.

Die verschiedenen Keynotes hatten keinen direkten Bezug zum Studium oder zur täglichen Arbeit in der Bibliothek, dennoch war es durchaus spannend, z. B. etwas über die visuelle Wahrnehmung beim Lesen und die Sprachverarbeitung oder über den Wert des Lesens und die damit verbundenen Rituale oder Praktiken zu erfahren. Besonders die Ausführung zur Selbstdarstellung in sozialen Netzwerken, wie Instagram, in Verbindung mit dem Lesen, war durch die lockere Vortragsart sehr unterhaltsam und brachte einige Zuhörer zum Lächeln - denn so mancher Studierende erkannte sich selbst wieder. Neben den Keynotes der Wissenschaftler deckten die Paper-Sessions der Studierenden und Young Professionals (Bachelor- und Master-Studierende - hauptsächlich aus Europa) eine ganze Bandbreite an Themen in Kurzvorträgen ab. Dort wurde u. a. vom Digital Storytelling in öffentlichen Bibliotheken, von Gamification in Estland, über die Digitalisierungsschwerpunkte in unterschiedlichen europäischen Regionen und ebenso von social media-affinen Universitätsbibliotheken und deren Facebook- und Instagram-Accounts in Estland berichtet.

Zum Mitmachen regten die unterschiedlichsten Workshops an. Hier wurden zum Beispiel anhand unterschiedlicher Indikatoren Fake News ermittelt, Social Media-Pläne für Bibliotheken und andere Gedächtnisinstitutionen erstellt, Social Justice (oder das Gegenteil davon) ermittelt und vieles mehr. Die Inhalte der Workshops konnten meist direkt auf Studieninhalte oder den Arbeitsalltag angewendet werden. Exemplarisch werden hier drei davon kurz vorgestellt:
Unter dem Motto „How to trust the internet again?“ wurde ein Leitfaden zur Identifikation von Fake News vermittelt, den Studierende bei der bibliothekarischen Vermittlung von Informations- und Medienkompetenz bei Benutzungsschulungen und an der Informationstheke verwenden können.

Bei „That's been noted! - A workshop on students current note-taking behaviour, motivation and future perspectives in a digital learning environment" gab es hilfreiche Hinweise zum eigenen Verhalten im Studium. Wie erstellt man selbst Notizen? Was macht sinnvolle Notizen aus und wie werden sich analoge und digitale Notizen zukünftig weiterentwickeln?

Auch der Workshop „To be or not to be - it's not the question about library social media marketing!" war bereichernd, da man sofort ausprobieren konnte, wie in den sozialen Netzwerken Facebook und Instagram erfolgreich Posts mit hoher Reichweite veröffentlicht werden können. Dies ist heutzutage aus Bibliothekssicht besonders wichtig, um Studierende und andere Nutzer effektiv zu erreichen, da fast jede Bibliothek mindestens einen Account in einem der vielen sozialen Netzwerke besitzt.

Außerdem gab es eine Poster-Session und - dieses Jahr erstmals bei der BOBCATSSS - das Kurzvortragskonzept PechaKucha, ein kurzer Vortrag mit 20 Folien à 20 Sekunden, der einen kurzen und informativen Einblick in verschiedene Themen geben kann. Bei den Postern hatten die US-Amerikaner zahlentechnisch die Nase weit vorn. Posterthemen waren beispielsweise Harry Potters Einfluss auf das Leseverhalten einer ganzen Generation, die Einrichtung von Gefängnisbibliotheken (so wurde auf Bibliotheksmöbel hingewiesen, an denen Menschen festgekettet werden können!), welches Verhalten Studierende beim Aufenthalt in Universitätsbibliotheken aufweisen, ein Projekt zur literarischen Aufarbeitung der russischen Revolution und, und, und ...

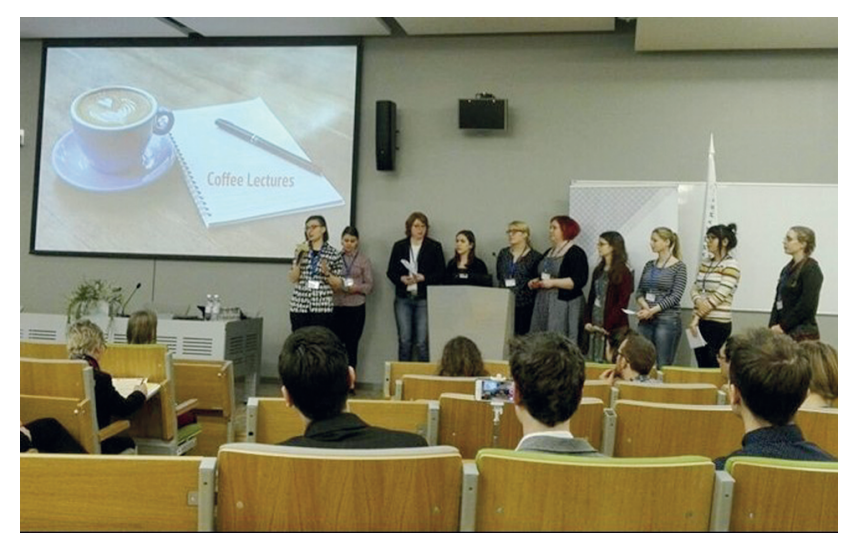

Abbildung 2: Pecha-Kucha-Beitrag. (Foto: Silke Clausing) 
Die zehn Studierenden aus Hannover steuerten einen Pecha-Kucha-Beitrag zum weiten Thema Informationskompetenz bei und berichteten von Hochschulveranstaltungen, Projekten und praktischen Beispielen aus deutschen Bibliotheken, wie z. B. Coffee Lectures.

Neben dem Konferenz-Programm sowie den Abendveranstaltungen wie Konferenzdinner und -party war es möglich, Riga und seine Bibliothekslandschaft kennenzulernen. Die neu erbaute beeindruckende Nationalbibliothek oder die 24-Stunden-Universitätsbibliothek haben architektonisch einen bleibenden Eindruck hinterlassen. Die eine oder andere Einrichtungsidee (wie z. B. Sitzsäcke oder Schaukelstühle) könnte gerne von deutschen Bibliotheken übernommen werden.

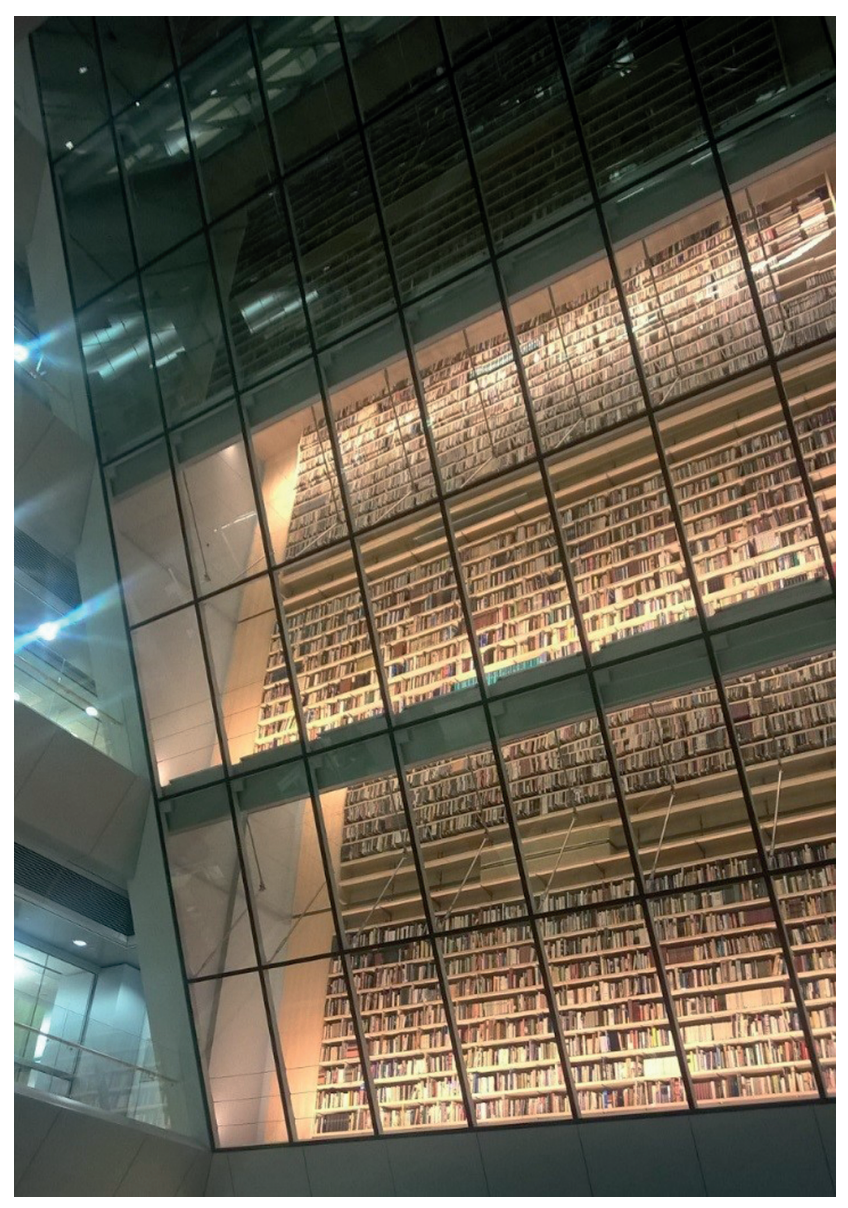

Abbildung 3: The Bookshelf of the Nation im Atrium der Nationalbibliothek. (Foto: Ina Blümel)

Trotz des Vormarsches von jungen Formaten wie Barcamps, Hands-On- oder Project-Labs andernorts orientiert sich die BOBCATSSS nach wie vor an klassischen Konferenzformaten. Ob das immer so bleiben muss, sei dahingestellt. Mit in diesem Jahr um die 240 Teilnehmenden geht es wesentlich familiärer zu als in den Anfangsjahren der BOBCATSSS. Grund für sinkende Teilnehmerzahlen sind sicherlich themenfokussiertere LIS- und IKT-Veranstaltungen, insbesondere dann wenn diese mit renommierten Keynote-Speakern besetzt sind. Das Motto der diesjährigen Konferenz „The Power of Reading” ließ viel Freiraum, sodass der inhaltliche Bezug mancher Sessions nicht deutlich wurde. Hier hätte man sich eine klare thematische Begrenzung gewünscht. Für eine ausschließlich von Studierenden geplante Konferenz, gab es bei dieser BOBCATSSS organisatorisch aber kaum etwas zu bemängeln. Änderungen im Programm wurden rechtzeitig mitgeteilt und auch die Zeiten der einzelnen Sessions eingehalten. Die Moderation wies gut auf die nächsten anstehenden Programmpunkte wie Social Events hin, und nur am letzten Konferenztag fielen einige Sessions aus. Dafür wäre ein Ersatzprogramm schön gewesen. Beim Gala Dinner am Abend des ersten Konferenztages fehlten soziale Programmpunkte, bei denen ein Austausch über die Tische hinweg zustande hätte kommen können und so blieb es meist bei der Kommunikation mit den Sitznachbarn. Ein Mehrwert für kommende Konferenzen wäre eine Session, bei der die Studierenden, die zum Planungskomitee gehören, dem Publikum einen Blick hinter die Kulissen gewähren. Nachfolgende Organisatoren könnten davon profitieren und die Teilnehmenden lernen die Ansprechpartner kennen.

Die BOBCATSSS ist in erster Linie ein Format von Studierenden für Studierende. Dies hat den Vorteil, dass Ideen und Konzepte „frisch von der Uni” kommen. Themen und Tiefe sind heterogen wie die europäische LISAusbildungen zahlreich, und geben einen Einblick in und einen Ausblick auf die Zukunft des Berufsfeldes. Auch wenn sich die jeweils ausrichtenden Hochschulen gemeinsam mit EUCLID bemühen, der Vielfalt eine jährliche thematische Klammer zu geben, steht die Konferenz nicht für fachliche Exzellenz in einzelnen LIS-Themen. Stattdessen liegt der Fokus auf dem Austausch mit internationalen Studierenden über die unterschiedlichen Ausprägungen und Inhalte von Library Information Science und findet in dieser Form wohl kaum bei einer anderen Veranstaltung statt. So ist die BOBCATSSS für LIS-Studierende eine der besten Möglichkeiten, mit vielen Studierenden anderer inund ausländischer Hochschulen in Kontakt zu treten.

Insgesamt ist der Besuch der BOBCATSSS für Studierende und Young Professionals sehr zu empfehlen. Es ist eine einmalige Gelegenheit, über den Tellerrand des deutschen Bibliotheks- und Informationswesens hinauszublicken. Die Studierenden der Hochschule Hannover empfanden den Besuch der BOBCATSSS 2018 als sehr bereichernd für ihr Studium und ihre zukünftige Berufspraxis. Sie profitierten sehr von der Erarbeitung und Präsentation eines 


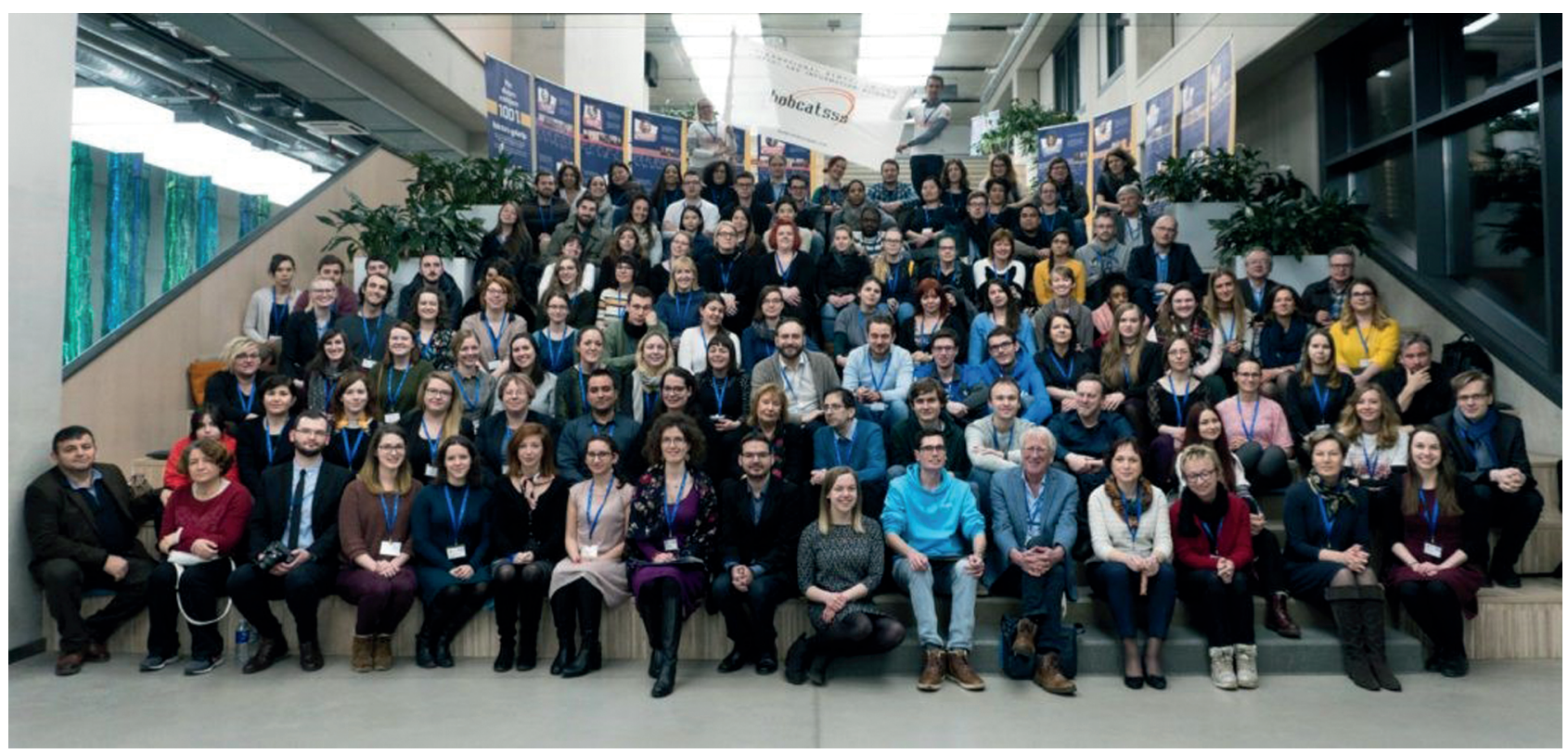

Abbildung 4: Gruppenfoto BOBCATSSS 2018. (Quelle: https://bobcatsss2018.lu.lv/files/2018/02/BOBCATSSS-2018-family-all-together1024x485.jpg)

Kurz-Vortrags vor Fachpublikum und bedanken sich für die Finanzierung der Exkursion bei der Fakultät III-Medien, Information und Design.

Deskriptoren: Veranstaltung, Tagung, Tagungsbericht, Bibliothekswissenschaft, Informationsmanagement, Informationswissenschaft, BOBCATSSS

\section{Dr. Ina Blümel}

ina.bluemel@hs-hannover.de

Silke Clausing

silke.clausing@hs-hannover.de

Franziska Altemeier

franziska.altemeier@stud.hs-hannover.de

Amelie Andresen

amelie.andresen@stud.hs-hannover.de

\section{Aline Brun}

aline.brun@stud.hs-hannover.de

\section{Carina Dony}

carina.dony@stud.hs-hannover.de

Marisa Kaminski

marisa.kaminski@stud.hs-hannover.de

Antje Pellowski

antje.pellowski@stud.hs-hannover.de

Helena Schugt

helena.schugt@stud.hs-hannover.de

Anne Talk

anne.talk@stud.hs-hannover.de 